\title{
Tele-operation and Human Robots Interactions
}

\author{
Ryad. CHELLALI \\ Human Robots Medited Interactions Lab. Tele-robotics and Applications Dept \\ Italian Institute of Technology \\ Italy
}

\section{Introduction}

We are daily and continuously interacting with machines and so-called 'intelligent' manmade entities. We push buttons and we read instructions to get money from cashdispensers, we tune the washing machine or microwave oven with more or less efforts quasi-every day. Following that, one can easily admit that our era is heavily based on manmachines interactions and the easiness one has in handling such machines is capital, mainly in terms economical, social and psychological impacts. Robots, as a singular sub-set of these machines, are also subject to the same constraints and preoccupations. Moreover and unlikely to mobile phones, PDA or other intelligent devices, interactions with or through robots (tele-operation scheme) are more critical and more specific: interactions with robots are critical because robots are designed to achieve complex tasks within versatile, changing and hazardous environments. They are specific because robots are used instead (sometimes as extensions) of humans (for safety or for economical reasons) leading to confusions between machine-robot and living-robot concepts.

The objective robot (the machine executing a program) and the subjective robot (the anthropomorphic robot and its image in folks mind) are entities too complex to be seen only as simple input-output black boxes. We believe that interactions with and through robots need very advanced and multi-disciplinary methodologies for designing human-robots communication, co-operation and collaboration interfaces.

In this chapter, we give our vision for human-robots interactions. For this purpose, we propose to revisit the robotics timeline. We will show through this timeline the strong relations between robotics and tele-operation. These relations will be depicted under two perspectives: firstly, from human-robots interactions point of view and then from robots autonomy one. The natural and effective junction between these two visions will take place with the companion robot, e.g. the autonomous robot which is able to co-operate and to collaborate with humans. We belive that before reaching this robotics' ultimate goal, one must answer to a central problem: how humans perceive robots? This formultaion and the answers one can give to the question will undoubtly lead to design effective robots and simplified tools allwoing natural and transparent human-robts intercations.

The document is organized as follow: the first part gives some historical hints letting the reader have a synthetic view of robotics' story. In the second part, we develop our theory about human robots interactions. We will see how we can built a new framework, namely 
the anthropomorphic robotics, by combining existing theories coming from neuroscience, psychology and psycho-physics. We show then that this theory can support simple teleoperation problems (delays, cognitive overloads, physical distance, etc.) as well as advanced human-robots co-operation issues.

We finish by presenting some tools we are developing and some exemples of researches we are conucting to assess our hypothesis.

\section{A brief Robotics history}

In this part we discuss robotics' history. This last has a lot of versions, containing myths, lies and realities. The purpose here is not to establish the exact history; historians will do this work better than us. The idea is to focus on the robotics time line in order to understand what the main motivations in robots development were.

\subsection{The imaginary robotics and the pre-robotics era}

Robotics historians agree that the first public use of the word robot was around 1921: it was introduced by the Czech writer Čapek in his R.U.R (Rossum's Universal Robots) play to describe are artificial people. This factual reference came after many other official and unofficial histories of robots or what can be assimilated to robots. Indeed and as far as traces exist, the existence of artificial and human-like beings obeying and executing all humans aims and desires or behaving like them was an essential part of the folk belief. Such mythical characters were largely present and written stories exist for the Greek era (Ulysses et Talos for instance). A more practical idea and a tangible entity were proposed by Ctesibus (270BC). He built a system based on water-clocks with moveable figures. Al Jaziri in the $12^{\text {th }}$ century, proposed a more sophisticated set for the Egyptian emperor: he developed a boat with automatic musician including drummers, a harpist and flautist to entertain the court and the emperor's suite. In Japan during the same period, Konjaku Monogatari shu writings reported a mechanical irrigation doll. These developments were transferred to Europe via Frederic II who received a sophisticated clock from the Egyptian emperor's in 1232. Horology techniques hence received were developed and important new realizations were achieved: Leonardo Da Vinci, for instance, proposed an animated duck in the $16^{\text {th }}$ century and Pascal who built the first computer (Pascaline 1645). Jacques de VAUCANSON developed an eating, digesting and defecating duck, which can flap wings also. Many other examples followed during the Enlightenment-era like the 'La Joyeuse de Tympanon' music player offered to the French queen Marie-Antoinette. These efforts were continued and a lot of automaton like chess players, writers, animals, etc was created in Europe thanks to the mechanist stream. This last was not only used extensively to design and build improbable creatures, but also and mainly in industrial applications: De VAUCANSON for instance was also a lot involved in textile industry development in the area of Lyon in FRANCE. show their power through technical capabilities.

Another step was achieved in the 19th century: Frankenstein fiction creature (in 1818) was presented within a movie. Conversely to what was developed before, Frankenstein creation corresponds to a new vision and a new challenge and the movie suggested that humans can create living (in the biological way) entities. One can imagine that the purpose of this movie was to show that humans have enough knowledge to replicate biologically themselves, at 
least through their imagination and images and tendency still exists and movies like 'Terminator', 'AI', etc. had great successes the last decade.

In the 30's Asimov emitted his famous rules. Even if real robots did not exist, Asimov had formalized the ethical rules that may govern the relationships between humans and probable robots. His assumptions were purely imaginary and based only on supposed future robots.

The concept of robot perhaps exists since a long time. For sure not having the same meaning as we have it in 2009 but as an imaginary entity able to behave like humans and having an external biologically plausible shape. This entity exists already in the folk's mind that was shaped through mystic and mythological representations in the early times, mechanical during Enlightenment-era, virtual very recently and present today under humanoids or animats umbrella. The other interesting fact is that robots have served as a sign of power, successively mystic, military-industrial and technological.

\subsection{Tele-manipulation and Tele-operation to answer to real needs}

Since prehistory, humans developed tools to ease fundamental daily life tasks namely, eating, hunting and fighting (homo-habilis). To catch a pray or to cook it, humans used very early tools allowing to achieve the previous vital tasks. When considering cooking, humans utilized sticks to avoid to be burned. This behavior can be seen as the first transfer of dexterity at a distance of some cm's and can be considered as the ancestral tele-operation. Closer to us in the 40 's, the need of manipulating dangerous products, mainly nuclear substances appeared to be essential for military applications. This leaded to the construction of the first tele-manipulators. R. Goertz and his group developed at ANL a set of prototypes (E1 to E4) of mechanical-based remote manipulators. These researches were done at that time to give operational solutions to immediate and sensitive problems the nuclear industry was facing. The first systems were passive, i.e. tele-manipulators were based on mechanical systems allowing to human forces and efforts to be transmitted to a slave. It is obvious that for these systems both energy and decision making were completely handled by the operator. Thus, one can easily imagine physical and mental operator's heavy workload, leading to a fatigue limiting performances. A first improvement was done by introducing energy into the system. Electrical actuators were used to supply user's forces. sensors and controllers. In such way remotely controlled manipulations were simplified by injecting energy to the system and by discharging operators from low level controls. The further developments of tele-operation were concerned with the introduction of more 'intelligence' within the system. Indeed, thanks to the advances made in computer technology and automatic control theory, some aids were introduced to help the tele-operator and to discharge him from low level tasks. All was done to ease the process to human operators and let them manipulate distantly and dexterously dangerous and toxic products. However, the golden age of tele-operation was supposed to be finished in the beginning of the 60 's with the industrial use of the first autonomous manipulators.

\subsection{From industrial manipulators to mobile robots}

In the 50's and, the industry growth was huge and needs in terms technologies allowing more productivity and lower costs were a priority. Within this context, G. Devol and J. Engelberger decided to create Unimation, the first robots manufacturer. The purpose of the 
Unimation robots was to perform spot welding and any other task being hateful to workers. One can notice that these robots were derived from the ANL prototypes: both technological components and morphology are coming from Goertz prototypes. A short way to describe these robots is to consider it as a tele-manipulator where the operator was removed. Somehow, Unimation showed the way for developing robots' autonomy by integrating automation technology. This early introduction of the UNIMATE robot within General Motors and Ford chains was not economically sustainable, its Return On Investment (ROI) was very low compared to the one usually obtained through classical techniques (e.g. manpower). However, the psychological impact on US workers, US customers and more generally on worldwide population was much more than expected. Indeed, as people associates robots with myths and the most technological advances. In cold war and in an economical boom contexts, robots can show to others the USA power and to US customers that their products are perfect.

The next major step for robotics was made in the 70's. The boom of computers and the birth of computer science as an important research topic pushed researchers to look for visible and tangible applications. Indeed, Artificial Intelligence was considered as the ultimate finding and AI techniques were presented as tools able to compete with humans in terms of problem solving [24]. Robot manipulators were used as first demonstrators but it was not enough: the manipulator is equivalent to human hand only, no mobility neither consequent requirement could be tested. To go further and to proof Herbert predictions, the SRI proposed the first autonomous wheeled mobile robot [23]. This last was designed to deal with unknown environment by navigating, avoiding obstacles and recognizing objects of interest. This stream was fully supported by Artificial Intelligence school and served as a proof of AI capabilities [Minsky, McArthy]. For robotics community, these developments can be interpreted as the first efforts in increasing robots mobility, .i.e., transforming the manipulators into mobile platforms to face a wider world. This additional mobility the necessary integration of new aspect of robotics such as perception and locomotion. By doing so, e.g., by developing autonomy and locomotion, it was possible for robots to enlarge their working space from the assembly cell to wider and more complex environments.

Unfortunately, 30 years later the problem of autonomy was unsolved. [2] with his famous 'Elephants do not play chess' and many others [26] gave some explanations to AI failure. Nevertheless, many major advances have been achieved. Actuation and mobility were largely visited (mainly automatic control and sensors technologies) and very efficient solutions are existing actually. Indeed, efficient mechanical structures were built, from small bugs to humanoids to address locomotion. Moreover, interesting solutions were proposed letting robots walk, fly and swim with high accuracy, large mobility and stability. As well, computational and sensing capabilities have been also improved to a fascinating level. These technological solutions enabled to handle many aspects, mainly, low level controls which could be considered as solved. Unfortunately and despite all efforts and technological developments, decision making and autonomy are remaining bottlenecks.

\subsection{Humanoid robotics}

In parallel to the SRI-SHAKEY project, the University of Waseda launched the project Wabot-1 (1970). The aim of the project was to develop the first full scale human-like robot. It was presented in 1973 as the first anthropomorphic robot. This was a major jump for robotics: the idea behind was not to serve only as a test-bed for Artificial Intelligence 
developments but also to focus on its main property: the anthropomorphism. This last characteristic or property was considered as the necessary condition for robots to be accepted as companions. Indeed, the Wabot- 1 was targeting the field of human assistant or human companion. It appeared early that the necessary condition to achieve such a goal was its human-like shape: the robot needed to be designed as a human. This morphology was supposed to be the logical way to allow the robot to evolve in the same indoors environments and to satisfy all the consequent (i.e. man made environments) constraints. Another motivation for shaping the robot this way was perhaps the facilitation of the human-robot interactions. The last motivation is a pure and personal speculative interpretation: by creating such a robot, the developers were targeting high emotional, psychological and economical impacts, e.g. it was a way to show that the Japanese industry is on his way to master and to reach the top edge of technology as it was done earlier in USA with manipulators.

The humanoid robotics was there and researchers faced more complex problems than for wheeled platforms. In all sectors, the humanoid presented the most challenging issues: for motion control, for environment understanding and perception, for interactions with humans, etc. the humanoid requested more advanced solutions. One of the first efforts was dealing with locomotion. This issue is specific to legged robots and conversely to wheeled robots, legged robots in general and bipeds in particular need a dynamic stabilization while walking. This inherent issue was addressed early and the ZMP [4] formulation was proposed and many automatic control based solutions were proposed and implemented within this framework. More recently, bio-inspired approaches (CPG's for instance [27]) were proposed and implemented. From the mechanical point of view, specific solutions were developed to ease the stabilization, mainly bio-inspired structures and parts (including compliances, stiffness, etc.).

Perception was also a big issue and one of the most challenging topics. The first proposed solutions were directly derived from classical approaches. Computer vision-based techniques for instance were applied to navigation and object recognition. Likely, reasoning and cognitive researches adapted existing ones and transferred to the humanoid context.

Unfortunately these efforts were not sufficient and extra-robotics help was needed: the humanoid theme is considered nowadays as a transversal project where the cooperation of many disciplines (like neurosciences, bio-mechanics, nano-bio technologies, sociology, philosophy, psychology, etc) and fields in mandatory. Following that, important transversal initiatives through labs and institutions were launched [Icube project, HRP project, COG project, etc.].

However, we can notice the existence of two main streams targeting two different goals exist: the first one aim is to develop co-operative robots, e.g., having capabilities and abilities to understand human's desires, behavior, speech, etc having emotions and proper behavior, etc. The second stream uses humanoids as test-bed to better understand humans: the humanoid is used as a simulator to support human-based models assessments [25].

Humanoid robotics is in its early stages and the current work within the two previous streams can help us to learn more about us in order to derive therapies. Rehabilitation techniques and other prosthesis are part of the goals of humanoid robotics. It can help us also to design better interfaces in order to simplify and to ease the use of our daily life machines and tools [28]. 


\subsection{What can we learn from the past?}

From the previous brief history of robotics, one can derive some conclusions and some lessons. This may help to better understand the current status of robotics and to identify the targets of future researches.

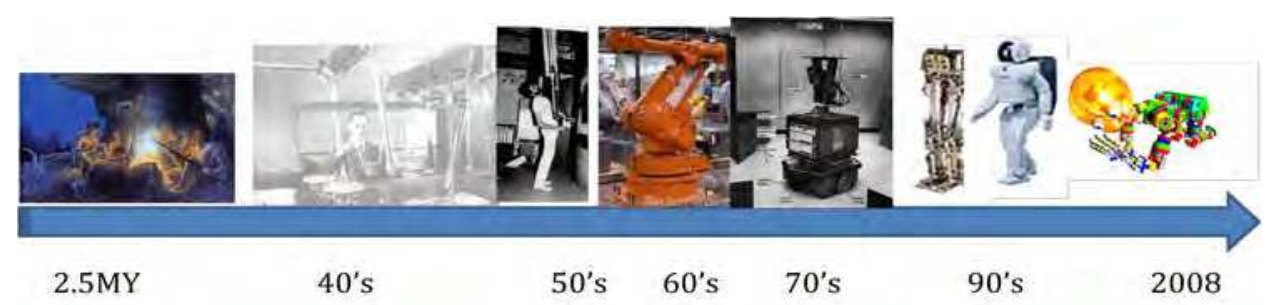

Fig. 1. Robotics timeline

The first observation one can make is concerning the singularity of the entity "robot". This last is a singularity and it is perceived as a particular entity; not an amorphous one and not a living one as well. This leads humans to develop very specific relationship with robots and there are differences between reactions people have when they interact with usual machines (washing machines, mobile phones, etc) and robots. Fundamentally these differences are small (except shapes and nature of functions they perform): both are materiel, they accept controls and orders execute programmed tasks and give feedbacks about their status. Does classifying a machine as a 'robot' changes its status? Does the shape play a role in this classification? And how this machine is then perceived by humans? The robot generates a lot of open questions which are still under investigation.

Some subjective explanations may be found in the pre-robotics era. Humanity belief at that time was largely influenced and shaped by myths of super manmade creatures. Later, the human-like automaton was no more a myth but a fascinating and tangible human creation. This fascination has probably created a specific status for these inanimate human-like entities and thus prepared the current perception of robots.

This last is subject these days to a lot of research and many efforts are done to qualify and to quantify this specific status. In other words, researchers are trying to determine how humans (and animals also) perceive robots. Social and emotional robotics shows that this perception is not unique and a lot of parameters are taken into account. Studies concerning robots' design and shape, embodiments in terms of animacy and intelligence, the age of users, etc have effects and impacts on human-robot interactions. One can imagine easily how the results can be used in the frame of companion and co-operative robotics.

However, these studies and the resulting hypotheses/theories may be moderated for teleoperated robots. Indeed, one may be aware that these last and autonomous robots have a fundamental difference: tele-operated robots have to achieve crucial, 'zero error' and measurable tasks while autonomous robots have more freedom and their errors are tolerated. Following that, the relationships between humans and the two categories of robots (e.g. autonomous and tele-operated) are different; in goal directed and 'zero error' interactions, human must adapt and must compensate the remote robot's limitations. For autonomous robots, the interaction is less constrained because the user is somehow less demanding. 
The third observation is more technical and concerns the modern robotics evolution. It makes sense to consider the first tele-operation systems as the ancestor of modern robots. As we said before, the robots' evolution was pushing developments and innovations toward autonomy. The timeline started with human-robot systems to move to systems where more 'intelligence' was included. Consequently, human presence was minored. However, robotics returned to root and to tele-operation whenever an operational system was needed: the presence of humans in the control loop was considered as the ultimate and safe solution.

The last observation is concerned with the contributions of the current tele-operation. This last is still improving human-robots interaction field. Tele-operation systems are the only ones putting in close and constrained contact robots and humans. This forced synergy pushes operators to adapt to machines, pushes engineers to find the best interfacing technologies and pushes researchers to understand human to build effective systems able achieve safely critical and vital tasks.

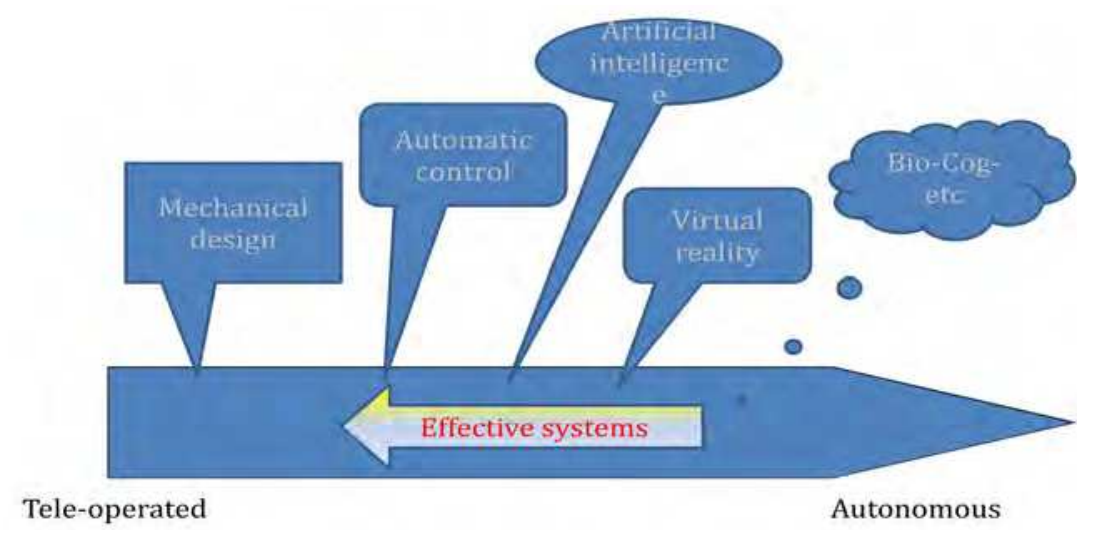

Fig. 2. contributions to robotics

\section{Tele-operation as a starting point to built a new vision of robotics}

In this part we propose to describe the current tele-operation systems. This will allow us to highlight the most important achievements, the advantages these systems and to finish, we will try to list their drawbacks and we discuss these last.

\subsection{Current Tele-operation systems}

First tele-operation objectives were to enable to control distantly complex mechanical systems, in order to (tele) manipulate dangerous and hazardous material. The first need at that time was concerned with dexterity: the system has to be enough dexterous to support simple tasks to pour some liquid into a bowl for instance. Four degrees of freedom mechanism (a pincer with a spherical link) was proposed to handle this class of tasks. Rapidly and after dexterity, other needs came-up. For safety reasons, the distance between the operator and the operation field had to be increased. This leaded to new needs, namely, a complex mechanical transmission was implemented. The first master-slave system developed by Goertz was purely mechanical and operators moved the slave through wires 
and tapes while having a direct view of the slave. By doing so, operators handled parasitic inertia and thus they produce additional workload not directly dedicated to manipulations. This distortion was corrected soon after. Indeed, electro-servomechanisms and controllers replaced wires and tapes. This allowed an electrical force reflecting position letting operators dedicate their energy to manipulations only.

After mobility, dexterity and force reflection improvements, people moved to ameliorate other sensing capabilities. Indeed and to protect users, a minimal distance between the master and the slave was imposed. Two problems rose with this mechanical separation: direct vision was no more possible and time delays appeared. For the visual feedback, simple live video streams were displayed on simple TV screens and for delays, people started by trying to understand its effects [11], namely they performed the first psychophysics studies to model human behaviour when performing tele-operation tasks. Their conclusions were that operators utilize the 'move and wait' strategy in presence of delay; humans compensate internally the closed loop delays. This leaded to the development of the so-called supervisory control; a heuristic approach where the controller allows the operator to specify tasks at a high-level. These tasks are decomposed by the controller into atomic commands and performed by the remote controller as a suite of simple tasks. The sequence is executed under the operator's supervision (.e.g. the operator can interrupt the process at any time; he can also modify the task's description content or level). This symbolic (or AI based) approach leaded to software solutions to provide more 'intelligence' and autonomy to the remote controller to compensate delays. A variation of this approach was proposed later with the notion of predictive displays [10].

This approach was followed by a huge effort from the automatic control community [29]. For the latter, tele-operation has been stated as a two folded problem: stability and transparency. For the first aspect, the goal is to maintain the stability of the system regardless of the behaviour of the operator or the environment. For the second, the goal is to allow tele-presence feeling, .e.g., hide the interface and let the operator perform interactions as he was within the remote environment. Many advances, mainly Lyapounov based analysis, impedance and hybrid representations, passivity based control schemes, etc., were made allowing stable and very efficient solutions to handle inherent delays like in space, underwater applications. Likely, transparency was tackled through the two ways transmission of force and velocity.

Nevertheless, force reflecting and visual feedback appeared very early as insufficient sensory modalities to guaranty efficient remote interactions: operators need more than $2 \mathrm{D}$ viewing and haptics-based links with the remote world. More sensing technologies and displaying devices were integrated or developed to improve existing systems in terms of immersion [11] [12], [17].

A lot of work has been done for instance on the visual channel. Sheridan summarized the influence of video feedback on tele-operator performances. Frame rate effects, resolution, colors, occlusions and position of the operator's point of view were also studied. It was shown that performances were affected. Haptics channel received also a lot of attention. Force feedback arms used are the typical and the most studied bilateral ways of controlling slave robots: it closes the loops of force-torque based interactions.

Many similar technical solutions were proposed for other channel, mainly tactile, auditory kinaesthetic and even olfactory. The guidelines for the design of such tools were directed toward reproducing as exactly as possible information, actions and reaction flows for both 
sides (the master and the slave): on one hand, the master (human operator's side) must capture all the desires of the human operator. On the other hand the slave must capture the "image" of the remote world and translate it into stimuli for the operator to let him fill itself within this world. This latter concept or definition is well-known as tele-presence. It was introduced in the late 80 's by S. Tachi [20] to describe systems allowing users to feel them self within remote worlds. Asymptotically, tele-presence systems are the ones enabling to humans operator to tele-exist, i.e., not only to feel being somewhere but also letting distant people feel the presence of the tele-existent person. In fact both systems are theoretical models and their practical implementation is limited because of technical and fundamental barriers.

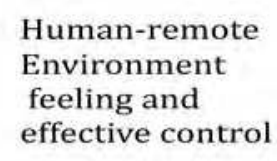

Fig. 3. toward tele-exitense

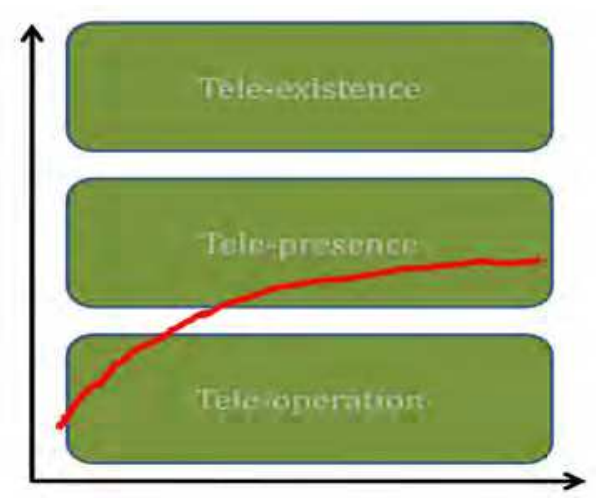

In parallel to the previously described technical efforts, some works tried to reconsider the tele-operation problem from the human-centred system point of view. Indeed, human is a central piece of master-slave systems: he issue commands function of what he perceives from the remote world. Following that, ergonomics and human factors appeared within tele-operation field and several studies were conducted. These latter were initially inspired and derived by previous works in man-machine and man-computer interfaces. The problem was stated as the handling of complex systems and sensory feedbacks and input devices were the identified key issues. The formulation was the following: to let people interact distantly, one needs to collect the maximum information about the remote world and display this information as soon as possible and as accurate as possible to operator. Likely, to transmit orders and controls, efforts were made to construct simplified and effective input tools.

\subsection{Current systems drawbacks}

In literature, tele-operation systems drawbacks are mainly identified as consequences of distortions or/and the absence of sensory feedbacks or/and the weak knowledge of the slave. This is partially true. The part of truth is due to technological limitations. It is easy to notice that current sensing, transduction and displaying technologies cannot reproduce stimuli (at all or at least partially) nor capture intentions that should be generated directly and not synthetically (e.g. through interfaces). 
Indeed, the current technology have access and can measure only few human parameters like gestures, speech, forces, torques, postures, direction of sight, etc.... Likely, to display the remote environment, current systems transmit incomplete and distorted information, like live video flows, forces, torques, sounds, etc... In both directions, information is partly missed or distorted. When controlling the slave, the operator compensates this lack of information for both sensory and motor aspects. He rebuilds mentally the remote space from the available feedback fragments. As well, he generates, the right slave's controls through the mental representation he has about the remote system. Said differently, the operator try

- To build a perfect matching between his space and the slave's one by compensating missing parts and by removing all the sensing and displaying based distortions.

- To build a model of the slave

The previous points could explain the cognitive overload situations and operators fatigue arising when remote interventions are performed or more generally when a human is piloting a complex system. This conclusion has to be reconsidered for tele-operation systems.

The other issue is more fundamental and it is concerned with unquantifiable parameters. Indeed, within tele-operation systems, the slave robot is a constructed as a machine with a very specific set of capabilities. However, people perceive it in a dual way: it can be seen as the classical tool that one can use to modify the environment or it can be seen as the semiautonomous entity obeying and accomplishing human commands and desires. In the first case the tool is considered as the operator's body extension, i.e., a mean to increase the personal working space. In the second case the slave is considered as an exogenous entity supporting human orders and informing him about its status and its environment. The two schemes and visions have different implications on the operator's sensory-motor system. On one side, the slave robot has to be integrated within the operator's sensory-motor space as part of his body. On the other side, one needs to build a mapping between two heterogeneous sensory-motor spaces.

Nevertheless, the two highlight the core problem, namely the existence of fundamental differences between the operator and the slave. These differences are the following:

- The difference of dimensionality between the operator's and the slave's sensorymotor spaces,

- The differences between stimuli perceived in direct interactions and the ones synthesized by the system's interfaces (a direct view and an HMD based display is a good illustration).

- The differences between the operator's physical actions (on the interface in this case) and the ones really achieved by the slave, e.g., the physical modifications of the remote world.

The previous differences reflect the distances tele-operation systems designers are trying to reduce. It overpasses the sole Euclidian space with his physical distances, time delays, scale changes, etc to include sensorial and motor spaces to create the optimum matching between the human and the robot spaces.

This formulation is just a new way to express the goals tele-operation systems' designers are aiming to reach, namely to reduce these differences or asymptotically to have the perfect tele-existence system. The main issues are still there because the human sensory-motor 
space is hard to describe and thus the metrics needed to operate on this space do not exist yet. People use intermediate spaces and indirect measurements, mainly derived from psychophysics, ergonomics and human factors, to assess or to design tele-operation systems. As said before, we have two working hypothesis: the system is considered as a body extension or as a semi-autonomous entity. Following the one or the other hypothesis, one needs to adopt a specific methodology to reduce the differences between the two sensorymotor spaces. In other words, the knowledge the operator must have and/or acquire about the slave is different. In the first case, the slave has to be integrated implicitly within the operator's sensory-motor system (e.g. considered as prosthesis). In the second one, the slave is considered as a collaborator with limited capabilities. Thus it requires the generation of specific controls and the development of specialized understanding skills. In both cases we can find a humanization aspect of the machine. This is specific to tele-operation and absent in other classical remotely controlled systems.

The last point, but not the least, is concerning the operator's sensory motor space. As presented before, this last is appearing like a classical vector space with linearly independent vectors set as basis (each corresponding to a sense or to a motor activity). This representation is missing an essential part, namely the cross-relations and the couplings between the sensory and the motoric components. In a hand-eye based action like catching for instance, any defect in one or other part influences greatly the other one. Researches taking into account the coupling effects started some years ago and they confirm the importance to reconsider the sensory-motor space construction and its use in the design of tele-operation systems. As a consequence of the previous finding is the following rule: motoric anthropomorphism is necessary but not sufficient to have an effective tele-operation system. An exoskeleton for instance cannot guaranty the efficiency of hand based interactions and other percepts (visual, tactile, kinesthetic, etc.) are needed.

To conclude and to open the next section, we can speculate on the ideal tele-operated system as oneself person: if one has its own image as a slave so he will make no efforts in controlling it and performing any kind of remote world transformation. The bijection between the remote and the master space and the mapping are perfect and no extra-efforts are needed to execute remote tasks. Somehow this is the asymptotic goal of tele-operation and amazingly humanoid robotics.

\subsection{Toward robot's autonomy and the necessity to have humans within the control loop}

Robots autonomy is one the first dreams of robotics' research. The pending and central question of robotics is the following: how to make a machine which is self-sustainable, e.g. able to move safely, to find its own energy, able to understand and to communicate with humans and other robots, etc. Many other capabilities can be added to this open list; dexterity in manipulating objects, recognizing these objects, understanding contexts and situations, etc.

This dream can be heavily moderated when having a look to autonomy definition or definitions. Indeed, we found plethory of definitions, each suggesting a singular and domain-dependent point of view. The most generic one is the following: "giving oneself' own laws". As expected, for robots and robotics, this definition is not fully true. Indeed, people program robots. By doing so, they transfer parts of their knowledge to robots. This knowledge is derived from the thoughts of the programmer and it reflects his answers to 
specific conditions (the task requirements). In other words, if a robot has to face a task, the programmer will imagine all the possible situations and consequently, all the suitable solutions to achieve the desired tasks. Certainly, learning, development and evolution procedures can increase robot's degree of autonomy, but formally, robots programming is equivalent to a priori tele-operation. One imagine a situation or a goal, derive the consequent robot behavior and the program it. This could lead the illusive autonomy and the robot will fail when facing unseen or unknown situations. This phenomenon can be illustrated through a parabola: the robot is put in a tunnel and the only way for him to evolve is to go back and forth without any chance to leave the tunnel, e.g. no way for the robot to find out an alternative to the imposed pathway. The robot's behavior is thus predictable and this is in contradiction with autonomy definition. Obstacle avoidance task is a good example for what I call illusive autonomy. Indeed, at a first glance, all obstacle avoidance behaviors implemetations are fascinating and could be considered as intelligent behaviors. In fact, the statement for this class of problems is mainly a measurement-based: robot sbuilds the geometry or the topology of the surroundings and adopt very simple algorithms to find out a free path.

Many other problems could be seen analogously as sensing-measurement problems (object recognition, localization, etc...) rather than advanced behaviors and real autonomy. Illusive autonomy is a consequence of designing biological like behaviors, acceptable for observers but without any justified foundations.

It appears that programming robots, namely transferring knowledge to robots is one of the key issues for building autonomous robots. We transfer methods and procedures, namely logical suites of actions hoping that it will cover all situations. One imposes partial predefined behavior and mechanisms to allow robots to handle situations we suppose them to be and to face.

The main question arising thus is the following: how to make such mechanism generic, e.g. the robot can learn new behavior without programming? As for children, robot cannot learn without the help of a more experienced entity (human or robot). The learning process needs examples and more than that, living examples.

Two sub-questions arise then: do we have the right hardware to support such mechanism and how to let the robot understand examples given by a more experienced entity.

The first sub-question is itself a research area and we will not address it here. Indeed, regarding hardware some functions are implementable others not: one cannot overpass the potential capabilities. Humans have a genetic potential leading to advanced behavior like adaptation. Animals for instance cannot overpass certain barriers: an herbivore cannot eat meat and become a carnivore even if its life is in danger. Changing alimentary regime is impossible (at least for short term horizons). A fish cannot run on the grass while humans can both swim and run on the grass. They adapt to learn swimming and more complex, they create specific tools to change their nature to go for instance underwater.

The second one leads to reconsider the human/robot robot interaction under the learningtransferring knowledge point of view. Tele-operation as the main field putting humans and robots together to achieve physical interactions may be a good candidate toward autonomous robots. On the other hand, if we assume that we have already autonomous robots, these last are supposed to interact with humans. Here also, a revisited tele-operation may play a major role to facilitate humans-robots communication [robonaut and tanie]. 
In this part, we proposed a new point of view from which tele-operation may be seen. In addition to be the tool of modifying physically remote, distant or inaccessible worlds, teleoperation also:

- Can help to design the right interaction paradigm between robots and humans,

- Could be an alternative solution to support the design of autonomous robots,

- Could be a tool to better understand humans.

\section{The anthropomorphic robotics for a new formulation of tele-operation}

The mechanical anthropomorphism introduced as the necessary but not sufficient condition to simplify the human robot communication and control. It simplifies the matching process between the human and the robot motor sub-spaces and thus allowing effortless control. The anthropomorphism I want to introduce here is a generalization and concerns the whole sensory-motor system. This generalization is purely speculative but it can be supported by a strong background and can be used as a framework to design efficient tele-operation systems and more generally, efficient interfaces. To do so, I rely on two existing findings in psychology and neurophysiology fields:

- The empathy and more specifically the perspective taking theory,

- The theory of mind and his neurological substrate, the mirror neurons.

\subsection{The empathy and the perspective taking}

1) The empathy

The concept of empathy appeared at the end of the 19th century within German philosophical circles. It was concerned mainly with human ability to "feel into" nature and man-made objects and the underlying question about the understanding of human aesthetic objects' appreciation. The central problem was to know why we perceive beautiful and ugly objects and how we use and sense data for our investigation of the world. Lipps extended the concept in the beginning of the $20^{\text {th }}$ century to overpass the aesthetic area. He claimed that empathy should be understood as the primary mean for our perception of other persons as minded creatures. At that time, this concept was the only alternative for conceiving of knowledge of other minds. It was described as a process with three steps that enable one to attribute mental states to other persons based on the observation of their physical behavior and one direct experience of mental states from the first person perspective.

- Another person $X$ manifests behavior of type $B$.

- In my own case, behavior of type $B$ is caused by mental state of type $M$.

- Since my and $X$ 's outward behavior of type $B$ is similar, it has to have similar inner mental causes. (It is thus assumed that I and the other persons are psychologically similar in the relevant sense.)

Therefore, the other person's behavior ( $X$ 's behavior) is caused by a mental state of type $\mathrm{M}$. This inference mechanism was largely used to explain social behavior of humans and the way they establish relationships. Nevertheless, this stream was criticized and abandoned to the theory theory approach (theory of knowledge acquisition, developmental phenomenon, learning mechanisms, etc.) which found his applications through AI. Empathy was considered as a very extremely naïve conception of human sciences to explain social relations, the influence of cultural context in human-human understanding, etc. 
For our purpose, empathy through the findings, the tools and the methodologies developed around this question in various areas like the human sciences, philosophy and more recently in neurosciences can be a good framework for tele-operation systems improvements. In other words, if the hypothesis of robots' and tele-robots' humanization is true, then human-human interactions knowledge can potentially transferred human robots interactions and recent works tends to demonstrate objectively the validity of this approach at least for humanoids[Krach].

\subsection{The perspective-taking}

More than for empathy, there no exact or unique definition of perspective-taking: it is research field dependant. If we consider for our needs and our purposes, we will consider its materialist side and somehow a geometrical point of view of empathy. We can define it as the ability one has to drift in and out of his point of view and how this drifting leads to the building of the so-called 'god's eyes view' [30]; If I'm at someone else place then I can feel what he feels and thus I can understand him.

Indeed, in one of its versions, the perspective taking theory was concerned with spatial cognition [31]. Following Berthoz [19], the spatialialization or perspective-taking allows one shift from a world's egocentric point to view to an allocentric one. This process is considered as an essential process and one the main components of empathy: it materializes and it describes objectively the way we can take others points of view at least to experience their surroundings.

For tele-operation the consequence is immediate: do tele-operators project themselves on the remote robot and construct a remote point of view to achieve physical interactions? A lot of experiments concerning this topic are in progress and partial and indirect answers to this question through experiments are already found. However, is still an open question to be developed in the next few years.

\subsection{The mirror neurons and neurosciences}

Nowadays Empathy is back. The first revival occurred in the 80 's with the simulation theory. This theory conceives ordinary mindreading as an egocentric method where one uses itself as a model to simulate other people's state. More recently and thanks to important findings of neurosciences, empathy can be again considered as an interesting framework to explain how a human recognizes another person's emotional states and intentions. Indeed, empathy received some empirical confirmation through mirror neurons. Neuroscientists have shown that there is a significant overlap between neural areas that underlie our observation of another person's action and areas that are stimulated when we execute the very same action [32]. In other words, they discovered that same brain areas are activated both when a motor activity is observed and performed. Likely, they showed also that humans simulate the motor activity within the mirror neurons area before executing it. This last argument is to add to those of the simulation theory defenders and as a contribution to the rehabilitation of empathy at least as a sustainable framework to explain low level interactions-relations take place.

The empathy together with mirror neurons research is very active. A lot of issues are still pending and no evident proof has been found to explain in detail the underlying processes. However, a lot of other areas are taking advantage of this formalism and apply it to several 
domains mainly in psychotherapy, education, art, business and economy, etc. Recently, some researchers investigated the extension of empathy to non-human beings. The question was to see whether or not humans can develop empathy toward animals, namely pets. The results are very surprising and might open new perspectives. Indeed, we just have formalism and some experimental evidences. The exact mechanism is not well known and practical and conceptual questions like the following are still open:

1- Can we have empathy with other biological systems?,

2- What information can human extract from seeing partial information about other humans?,

3- Do we interact better if we are manipulating something equivalent to biological systems?

The previous section introduced very briefly a framework which could be very interesting to our purpose. Indeed, the natural question that one could have is concerned with the transfer of the empathy framework to non-minded creatures like robots. We have many ingredients like "simulation theory", "perspective taking", "projection in other's mind" letting some freedom to speculate and answer affirmatively.

\subsection{Does empathy with robots make sense?}

By essence, the empathy with robots is hard to define and hard to obtain. One is in presence of non-minded and non-biological entities. But before going further, let me tell you what the reaction I had with humanoid robots was. Years ago, a colleague of mine showed me his humanoid robots team playing soccer during a RoboCup contest. After some minutes, I laughed for no apparent reasons. I saw the video twice and I had the same reaction. The way the robots were kicking the ball reminded me my childhood with children (including myself) performing the same actions with naïve and exaggerated imperfections. Was my reaction strange? Anyway, it was for me a questioning situation. A similar situation occurred some months later when I presented a humanoid robot in an elementary school: I observed strange and also questioning reactions. The perception of humanoids and robots in general is a key issue that must be well understood. Basically, we use and we perceive unconsciously some cues and some features leading us to construct an image of the robot. The uncanny valley phenomenon is certainly one of the first experiments trying to elucidate human robot's perception. Solving this issue is fundamental because it may allow simplifying the interactions between robots and humans. Consequently and in the light of we said before, the framework of empathy can be valid in this quest.

For us, the approach may be gradual: one needs first to start with tele-operation systems. Once these systems understood, one can move and tentatively generalize the findings to autonomous systems. For tele-operation systems, the empathy framework can be used as a basis to perform experimental researches. Indeed, for those considered as body extensions and those considered as exogenous entities, the integration within the operator's body scheme respectively the simulation scheme (e.g. operators simulate the motor-activity before sending the corresponding controls to the slave) can be applied.

In addition to offering a well developed set of experimental procedures and methodologies, the approach we are proposing can be evaluated objectively. Indeed, one of the interests of the empathy is that it shows where to search the effects and how to measure it objectively. It is obvious that neither the brain model nor the interpretation of its signals are available 
these days, but it is the only way to measure direct effects and thus to avoid classical indirect psychophysics-based interpretations.

\section{Virtual Reality as a major tool for anthropomorphic robots assessment}

Virtual reality is nowadays a major component of tele-operation systems. The acquaintance and cross-fertilizations between the two are old [Coiffet]. More specifically, VR is largely used for both simulation and remote control. Indeed, in its simulation side, VR (and augmented reality) allows to operators to experience interactions with synthetic worlds by synthesizing stimulations (obtained from simulations of real sensors performing under real physical laws) for our main sensory channels like vision, auditory, tactile, haptic, kinesthetic, etc...

In its remote control version, the use VR/AR techniques is mainly dealing with sensory compensations, corrections or substitutions. Respectively, VR/AR systems recreate missing information, remove distortions and enhance sensory signals or perform transfers between senses (visual information is displayed as a tactile one for instance). That is said, VR/AR can be considered as a very flexible stimuli generator and one can address sensory channels with a wide variety of stimulations. This maturity is partly due to robotics and to teleoperation and their strong needs of operational and robust systems. This obliged VR people to find out new interfacing solutions covering the large spectrum of senses with high reliability and robustness.

Naturally and regarding the possibilities VR is offering, human centered researches (like cognitive sciences, psychology, neurosciences, etc) came to VR. These technologies are enough flexible and enough powerful to enable to these new demanding fields to setup new approaches and new experiments for understanding the human brain, its functions and the way it process/analyze external stimulations to built complex and powerful behavior. These systems can support infinity of scenarios addressing all human senses in a cost-effective way.

Following that, VR is the best candidate to support the assessment of the empathy-based framework and its translation into the anthropomorphic robotics hypothesis. Following that, we started to perform some experiments but we rapidly faced an unexpected problem: VR is a set of tools and not a science. In our case, a problem concerning depth perception raised when we performed experiments dealing with interactions within unfamiliar environments (with unfamiliar physical laws like nano-spaces or micro-gravity spaces). We obtained biased results even if our visual displays were very well calibrated geometrically. The visual perception and specially depth perception within VR systems is still an open problem to be solved or at least to be well known to avoid biased use and incorrect analysis. For other senses the same conclusion is valid and especially when different modalities are combined.

\subsection{Is VR fully reliable: an example through depth perception}

VR is supposed to recreate real worlds by stimulating human senses according to natural laws. However, VR cannot generate all the possible stimulations one can perceive. On the other hand, for those possible, the stimulations are mostly distorted or incomplete. Following that, the use of VR is not as magic as it is said. One has to take care with it and to understand all the undesired phenomena VR can induce directly or indirectly. In the next 
lines, a specific and known problem is given to illustrate the fact that VR has to be more effective.

Immersive viewing devices are key elements for virtual reality systems. They address the richest sensory channel (supporting $80 \%$ of human sensory inputs) and thus, regarding the rendering quality, users may experience more or less realistic environments and interactions with these environments. Unfortunately, the above mentioned quality is depending on parameters which are not well understood. Namely, displaying devices affect both perception and actions in virtual environments in a hidden way. This leads to malfunctions and biases in sensitive applications like psychology research and therapy, training or education. Undoubtedly, absolute distance or depth perception is one the main issues and one of the most investigated topic in VR. Many research efforts have been performed to determine the effectiveness of different cues necessary to perceive depth.

For instance, many research reported a systematic underestimation of distances when HMD's are used compared to the same estimation in the real world. Many hypotheses were emitted to explain this phenomenon:

- the reduction of the field view effect,

- the weight of the HMD effect,

- the difference between the viewed world and the experimental place,

- etc.

Indeed, several studies on distance perception using the HMD have found significant underestimation of egocentric distance, the distance from an observer to a target. It was shown in that this underestimation is not due to the limited field of view of a user while using the HMD. In [18] for instance, it is argued that mechanical properties can play a role in the underestimation phenomena. Other explanations have been also given like lack of graphical based-realism or mismatches between the viewed world and the experimental environment (e.g. subjects are aware that the viewed scene does not correspond to the place where the experiment is performed). Likely, other works showed that other sources like visual cues (such as accommodation and convergence) or situations (visually directed actions) may also affect distance or depth estimations and thus decrease the feeling of immersion. In sum, the identification of sources leading to the distance misestimating effects for HMD's is still an open question. We verified the lacks found in literature. In our work we aim to verify the above mentioned phenomena. Our approach is based on the comparison of performances between HMD's and stereoscopic wide screens when simple verbal and relative depth estimation is achieved by seated subjects. By doing so, we simplify the experimental conditions and we concentrate only on few variables. Namely:

\subsection{Some examples of VR use in the context of tele-operation systems enhancement}

Hereafter, I give some examples of what VR can do. These examples are parts of our project dealing with tele-operation. The first one describes the experimental setup we are using to assess empathy with robots. The second one is an illustration of the possible derivations tele-operation can have.

2) Empathy measurement: a tentative experiment

Our goal with this experiment is to verify the hypothesis concerning the existence of an empathy-based relation between human and robots. 


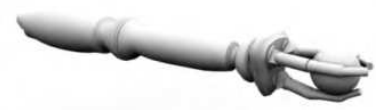

Fig. 4. Empathy measurements

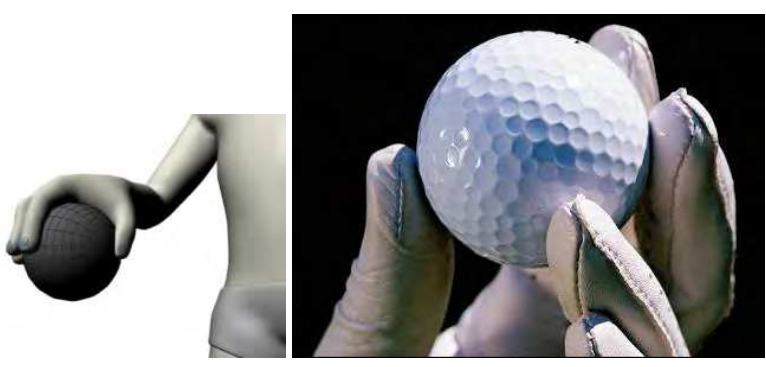

The setup we built is based on a set five hands. Four of them are synthetic with respectively $3,4,5$ and 7 fingers. The last one is realistic (a copy of a human hand). All of them are controlled by users through a data glove. We will not discuss here the experiment and the preliminary results we have but just to highlight the usefulness of VR in terms of flexibility: it allows us to enlarge our spectrum of potential stimulations to see how users project themselves on anthropomorphic and non-anthropomorphic structures including funny and unrealistic targets (a hand with 7 fingers).

3) Sensory substitution as a minimalist way to improve interactions

The second example is dealing with sensory substitution. The idea behind is to see how can we replace a sensory modality by another one. Namely, we want to study the effects of displaying geometrical information for navigation purposes through the tactile modality. One can imagine easily that for blind people such substitution is important.
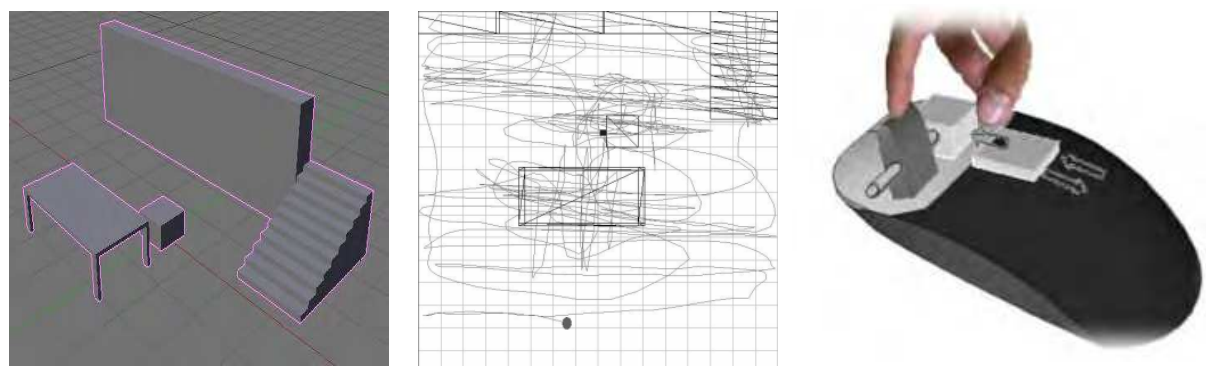

Fig. 5. DIGEYE project

The starting point of our work was purely dealing with tele-operation. We wanted to build a device to help navigation in environments where the visual information was not available. A prototype was achieved and tested. During these tests, we wanted to verify if people used with absence of visual information perform better than normal users. Following that, we decided to build a complete system allowing feeling the 3D geometry of the environment. We built this device including a stereo-vision system (for 3D modeling) and a haptic mouse with an actuated stick. The 3D geometry of a viewed scene is felt through the finger as a function of the mouse position and the stick height. We found that the information such device can display is not rich but enough for some tasks, namely, it allows to navigate safely. More recently and after brain activity signals analysis, we discovered that the device 
can be used as a diagnosis tool to diagnose some cognitive and brain pathologies. That is to say, our first problem, dealing with pure tele-operation issues leaded us to unexpected application.

\section{Conclusions}

In this document, I tried to give an overview of tele-operation: its history and origins, its background, its drawbacks and the perspectives it might offer to current researches and demands in robotics and other fields.

The construction of the future tele-operation/robotics is in progress and many people are still working on it. Surgery, prosthesis, rehabilitation, neurosciences, space, underwater and many other domains use this technology and the effort must be continuous.

The ideas described in this document are now facing the field reality through the experiments we are conducting. The preliminary results are encouraging, but a lot of work and a lot of efforts are necessary to progress.

I believe that tele-operation can help. However it cannot progress alone, it is necessary to work in multi-disciplinary way: tele-operation needs all the available knowledge, techniques and methods dealing with humans. We believe that technology alone cannot provide tools to achieve the robotics dream.

\section{References}

[1] Frédéric Kaplan. (2004). Les machines apprivoisées: Comprendre les robots de loisir Vuibert Ed, 2004.

[2] Rodney A. Brooks. (1990). Elephants Don't Play Chess Robotics and Autonomous Systems 6 (1990) 3-15

[3] Crevier, Daniel. (1993). AI: The Tumultuous Search for Artificial Intelligence, New York, Basic Books Edts, (1993).

[4]Vukobratovic, M. (1973). How to control artificial anthropomorphic systems. IEEE Trans. Sys. Man Cybernet. 3(5):497-507 (1973)

[5]Batya Friedman Peter H. Kahn, Jr. Jennifer Hagman. Hardware Companions? - What Online AIBO Discussion Forums Reveal about the Human-Robotic Relationship

[6]Erhan Oztop david w. Franklin, Thierry Chaminade. (2004). Human-humanoid interaction: is a humanoid robot perceived as a human? humanoids 2004

[7] Christoph Bartneck, Takayuki Kanda, Omar Mubin, Abdullah Al Mahmud. (2009). Does the Design of a Robot Influence Its Animacy and Perceived Intelligence? Int J Soc Robot (2009) 1: 195-204

[8] W. R. Ferrell. (1965). Remote Manipulation with Transmission Delay. IEEE Transactions on Human Factors in Electronics, 6:24-32, Sept 1965.

[9] Peter F. Hokayem Mark W. Spong. (2004). Bilateral Teleoperation: An Historical Survey Automatica 2004

[10] A. Bejczy, S. Venema, and W. Kim. (1990). "Role of computer graphics in space telerobotics: Preview and predictive displays," in Cooperative Intelligent Robotics in Space, pages 365-377, November 1990.

[11] T. B. Sheridan. (1993). "Space teleoperation through time delay: Review and prognosis," IEEE Transactions on Robotics and Automation, vol. 9, no. 5, October 1993. 
[12] S. Zhai, P. Milgram. (1991). A telerobotic virtual control system Proc. SPIE, vol. 1612, Cooperative Intelligent Robotics in Space ii, Boston, nov. 11-13, 1991.

[13] Sören Krach, Frank Hegel, Britta Wrede, Gerhard Sagerer, Ferdinand Binkofski, Tilo Kircher. (2008). Can Machines Think? Interaction and Perspective Taking with Robots Investigated via fMR. PlosOne-July 2008, (open-access at http://www.plosone.org/ article/info:doi/10.1371/journal.pone.0002597)

[14] Ackermann, E. (1996). Perspective-Taking and object Construction. In Constuctionism in Practice: Designing, Thinking, and Learning in a Digital World (Kafai, Y.,and Resnick, M., Eds.). Mahwah, New Jersey: Lawrence Erlbaum Associates. Part 1, Chap.2. pp. 25-37(1996).

[15] D. Weatherford. (1985). Representing and Manipulating Spatial Information from Different Environments: Models to Neighborhood. In: Cohen, R. (Ed.), The Development of Spatial Cognition, Lawrence Erlbaum, Hillsdale, NJ. pp. 41-70, 1985.

[16] R. Cohen and Cohen. (1985). The role of activity in spatial cognition. In: Cohen, R. (Ed.), The Development of Spatial Cognition, Lawrence Erlbaum, Hillsdale, NJ. pp. 199223. , 1985.

[17] Abderrahmane Kheddar. (2001). Teleoperation based on the hidden robot concept. IEEE Transactions on Systems, Man, and Cybernetics, Part A 31(1): 1-13 (2001)

[18] P. Willemsen, M. B. Colton, S. H. Creem-Regehr, and W. B. Thompson, (2009). The effects of head-mounted display mechanical properties and field of view on distance judgments in virtual environments, ACM Trans. Appl. Percept., vol. 6, no. 2, pp.1-14, 2009.

[19] Berthoz, A., Jorland, G. (2005). L'empathie, Eds Odile Jacob, 2005

[20] S. Tachi, H. Arai and T. Maeda. (1989). Tele-existence Visual Display for Remote Manipulation with a Realitime Sensation of Presence, Proceedings of the 20th International Syposium on Industrial Robots, pp.427-434, Tokyo, Japan (1989)

[21] P.Coiffet G.Burdea. (2003). Virtual Reality Technologies Wiley Eds (2nd edition), 2003

[22] Ee Sian Neo, Kazuhito Yokoi, Shuuji Kajita, Fumio Kanehiro, Kazuo Tanie: Whole body teleoperation of a humanoid robot -a method of integrating operator's intention and robot's autonomy. ICRA 2003: 1613-1619

[23] Hans Moravec. (1977). Towards Automatic Visual Obstacle Avoidance Proceedings of the 5th International Joint Conference on Artificial Intelligence, MIT, Cambridge, Mass., August 1977, p. 584

[24] S. Herbert. (1969). The science of the artificial, MIT Press, (1969)

[25] Metta G., Sandini G., Natale L., Craighero L., Fadiga L. (2006). Understanding mirror neurons: a bio-robotic approach Interaction Studies, 7(2), 197-232. 2006

[26] Pfeifer, R., Scheier, C., (2001). Understanding Intelligence (MIT Press, 2001)

[27] Ekeberg, Ö. (1993). A combined neuronal and mechanical model of fish swimming. Biological Cybernetics, 69, 363-374. 1993.

[28] H. Mayer, F. Gomez, D. Wierstra, I. Nagy, A. Knoll, and J. Schmidhuber. (2008). A System for Robotic Heart Surgery that Learns to Tie Knots Using Recurrent Neural Networks. Advanced Robotics, 22/13-14, p. 1521-1537, 2008

[29] R. J. Anderson and M. W. Spong. (1992). Asymptotic Stability for Force Reflecting Teleoperators with Time Delay The International Journal of Robotics Research.1992; 11: $135-149$ 
[30] Ackermann, E. (1996). Perspective-Taking and object Construction. In Constuctionism in Practice: Designing, Thinking, and Learning in a Digital World (Kafai, Y., and Resnick, M., Eds.). Mahwah, New Jersey: Lawrence Erlbaum Associates. Part 1, Chap. 2. pp. 25-37, (1996).

[31] Piaget, J. and Inhelder, B. (1967). The coordination of perspectives In The child's conception of space, Chap. 8. pp.209-246. New York: Norton \& Co. (1967)

[32] Fadiga, L.; Fogassi, L.; Pavesi, G.; Rizzolatti, G. (1995). Motor facilitation during action observation: a magnetic stimulation study Journal of Neurophysiology 73 (6): 26082611(1995), 


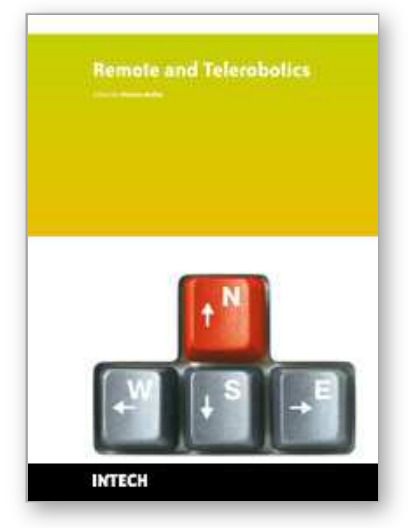

\author{
Remote and Telerobotics \\ Edited by Nicolas Mollet
}

ISBN 978-953-307-081-0

Hard cover, 220 pages

Publisher InTech

Published online 01, March, 2010

Published in print edition March, 2010

Any book which presents works about controlling distant robotics entities, namely the field of telerobotics, will propose advanced technics concerning time delay compensation, error handling, autonomous systems, secured and complex distant manipulations, etc. So does this new book, Remote and Telerobotics, which presents such state-of-the-art advanced solutions, allowing for instance to develop an open low-cost Robotics platform or to use very efficient prediction models to compensate latency. This edition is organized around eleven high-level chapters, presenting international research works coming from Japan, Korea, France, Italy, Spain, Greece and Netherlands.

\title{
How to reference
}

In order to correctly reference this scholarly work, feel free to copy and paste the following:

Ryad. Chellali (2010). Tele-operation and Human Robots Interactions, Remote and Telerobotics, Nicolas Mollet (Ed.), ISBN: 978-953-307-081-0, InTech, Available from: http://www.intechopen.com/books/remote-andtelerobotics/tele-operation-and-human-robots-interactions

\section{INTECH}

open science | open minds

\section{InTech Europe}

University Campus STeP Ri

Slavka Krautzeka 83/A

51000 Rijeka, Croatia

Phone: +385 (51) 770447

Fax: +385 (51) 686166

www.intechopen.com

\section{InTech China}

Unit 405, Office Block, Hotel Equatorial Shanghai

No.65, Yan An Road (West), Shanghai, 200040, China

中国上海市延安西路65号上海国际贵都大饭店办公楼 405 单元

Phone: +86-21-62489820

Fax: +86-21-62489821 
(C) 2010 The Author(s). Licensee IntechOpen. This chapter is distributed under the terms of the Creative Commons Attribution-NonCommercialShareAlike-3.0 License, which permits use, distribution and reproduction for non-commercial purposes, provided the original is properly cited and derivative works building on this content are distributed under the same license. 